\title{
SOME COMPUTATIONAL RESULTS ON THE SPECTRA OF GRAPHS
}

\author{
C. GODS IL AND B, MCKAY
}

The polynomial of a groph is the characteristic polynomial of its $0-1$ adjacency matrix. Two graphs are cospectral if their polynomials are the same.

In this paper some of the results from a numerical study of the polynomials of graphs are presented. The study has encompassed 9 point graphs, 9 point bipartite graphs, 14 point trees and 13 point forests. Also given are several theoretical results which were prompted by the numerical data. These include two characterizations of those cospectral grophs which have cospectral complements, and a proof that, in the sense of Schwenk [20] "almost no" trees are characterized by their polynomials together with the polynomials of their complements. In addition, mention is made of those cospectral graphs which have cospectral linegraphs, and those which are cospectral to their own complements.

\section{INTRODUCTION}

Graphs referred to in this paper have a finite, non-zero number of vertices and no loops or multiple edges. For such a graph $G, \bar{G}$ refers to the complement of $G$, and $L(G)$ to the linegraph of $G$. For brevity, a graph on $n$ vertices will be called an $n-$ graph.

Suppose $G$ is an $n$-graph. The adjacency matrix of $G$, also denoted $G$, is the $n \times n$ matrix whose $(i, j)$ th entry is the number of edges from vertex $i$ to vertex $j$. The polynomial of $G$, denoted $G(\lambda)$, is the characteristic polynomial of the adjacency matrix of $G$. An eigenvalue of $G$ is a root of $G(\lambda)$. The eigenvalues of $G$, together with their multiplicities, constitute the spectmon of $G$. Two graphs which have the same polynomial, and hence the same spectrum are called cospectral.

Other graph theoretic concepts not defined here can be found in Harary [9] or in Behzad and Chartrand [2]. For any square matrix A, the trace of A is denoted tr A. J will always refer to a square matrix with each element one and $I$ to an identity matrix.

The main purpose of this paper is to give the preliminary results of a computational study of the spectra of graphs. Previous studies of this kind have been made by Collatz and Singowitz [4] (5 point graphs and 8 point trees), king [13] (7 point graphs) and Mowshowitz [16] (10 point trees). In this study, the polynomials of 9 point graphs, 9 point bipartite graphs, 14 point trees and 13 point forests have been computed. Theoretical results which have been motivated by the numerical data include several characterisations of those cospectral graphs which have cospectral complements, 
and a proof that the proportion of trees of a given size which are characterized by their spectra plus the spectra of their complements goes to zero as the size increases strengthening a result of Schwenk [20]. Also considered are cospectral graphs' which have cospectral linegraphs, and graphs which are cospectral to their own complements.

\section{COSPECTRAL GRAPHS}

It has been known for some time [if] that the polynomial of a graph does not always determine the graph uniquely. The smallest example of non-isomorphic cospectral graphs is the pair of 5 -graphs shown in Figure 1. The smallest such connected pair is shown in Figure 2.
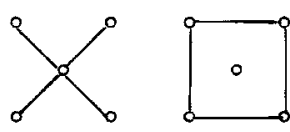

FIGURE 1
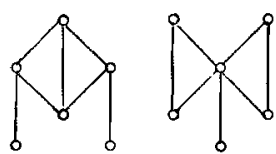

FIGURE 2

Despite some success in constructing large families of cospectral graphs [8], the problem of finding them all seems almost hopelessly difficult. Accordingly several computer searches for cospectral graphs have been made. The polynomials of the 7graphs were first computed by King and Read [11], [13], [17]. In this work the graphs with 8 or 9 vertices have been included. The source for these graphs was the tape distributed by Baker, Dewdney and Szilard [1]. The polynomials of the 274,668 ninegraphs were computed in about 50 minutes, using Danilevsky's method [3] programmed in assembly-language on a CDC Cyber 73.

In Table 1 (Appendix), the following data are given:

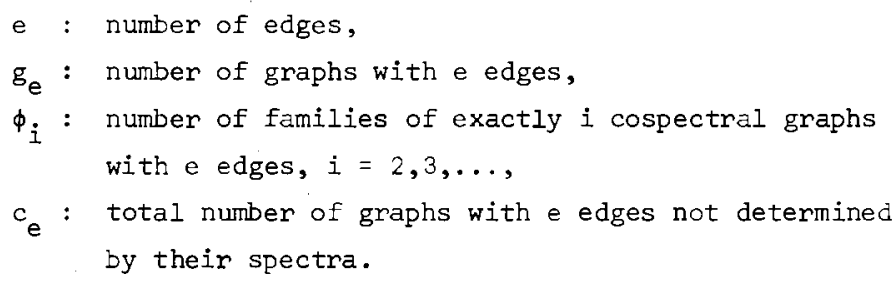

Values of e where $c_{e}=0$ have been omitted. Missing values of $g_{e}$ can be found in [9] or [10].

It is seen that about $18.6 \%$ of all the 9-graphs are not determined by their spectra and that this percentage appears to be increasing with the number of vertices. Unfortunately, the number of cospectral families is too large for them to be all listed here. However those with 7 or fewer vertices are given in Table 2. Graphs in the same row of the Table are cospectral. 
In Table 3 the extraordinary set of ten cospectral 9-graphs with 16 edges is listed. The equally large set with 20 edges is the set of complements of these graphs.

3. COSPECTRAL GRAFHS WITH COSPECTRAL COMPLEMENTS

In this section we consider the question of those cospectral graphs whose complements are also cospectral. The smallest of these are on 7 vertices, and are indicated by asterisks in Table 2. In Table 6 the statistical distribution of such families is presented in the same format as in Table 1 . Note that complementarity is a symmetric relationship, and so the lower half of each table has been omitted. It is perhaps surprising that about $86 \%$ of those 9-graphs not determined by their polynomials are still not determined when the polynomials of their complements are also considered. The following loose relationship probably provides only a partial explanation for this phenomenon.

Theorem 3.1 [5]. If the spectmon of a graph $G$ contains on eigenvalue $\lambda$ with multiplicity $\mathrm{P}(\mathrm{P}>1)$ then the spectmom of $\overline{\mathrm{G}}$ contains an eigenvalue $-\lambda-1$ with multiplicity $\overline{\mathrm{p}}$ satisfying $\mathrm{p}-1 \leq \overline{\mathrm{p}} \leq \mathrm{p}+1$.

We now proceed to characterize those cospectral graphs whose complements are also cospectral.

Consider each edge of a graph $G$ as two oppositely oriented directed edges. A walk of length $k(k \geqslant I)$ on $G$ is a sequence of directed edges $e_{1}, e_{2}, \ldots, e_{k}$ of $G$ such that consecutive edges in the sequence are adjacent. If $c_{k}$ is the number of such walks, for each $k$, then the formal power series

$$
W_{G}(x)=c_{1} x+c_{2} x^{2}+c_{3} x^{3}+\ldots
$$

is called the walk generating function for $G$. Note that we do not recognize walks of zero length.

Let $A$ and $B$ be arbitrary $n \times n$ matrices, and let $G$ be an $n$-graph. The following lemmas are stated without proof:

Lemma 3.2. A and B are cospertral iff

$$
\operatorname{tr} A^{r}=\operatorname{tr} B^{r} \quad r=1,2, \ldots, n-1
$$

Note that by the Cayley-Hamilton theorem [14], the upper bound on $r$ can be removed.

Lemma 3.3. $\operatorname{tr} \mathrm{AB}=\operatorname{tr} \mathrm{BA}$.

Lemma 3.4. (a) $\mathrm{J}^{\mathrm{k}}=\mathrm{n}^{\mathrm{k}-1} \mathrm{~J} \quad \mathrm{k}=1,2, \ldots$

where $\mathrm{J}$ is the $\mathrm{n} \times \mathrm{n}$ matrix with every element one.

(b) $\operatorname{tr} \mathrm{JAJB}=\operatorname{tr} \mathrm{JA}$ tr $\mathrm{JB}$. 
Lemina 3.5. $W_{G}(x)=c_{1} x+c_{2} x^{2}+\ldots$

where

$$
c_{k}=\sum_{i, j=1}^{n}\left(G^{k}\right)_{i j}=\operatorname{tr} J G^{k}=z^{T} G^{k} z, \quad k=1,2, \ldots
$$

where $z$ is an n-vector of all ones, and $\left(G^{k}\right)_{i j}$ is the $(i, j)$ th entry of $G^{k}$.

Theorem 3.6. Let $\mathrm{G}$ and $\mathrm{H}$ be cospectral n-grophs. Then $\overline{\mathrm{G}}$ and $\overline{\mathrm{H}}$ are cospectral iff $W_{G}(x)=W_{H}(x)$.

Proof. Clearly $\bar{G}(\lambda)=(J-G)(\lambda+1)$, so that by $3.2, \bar{G}$ and $\bar{H}$ are cospectral iff $\operatorname{tr}(\mathrm{J}-\mathrm{G})^{\mathrm{k}=\operatorname{tr}}(\mathrm{J}-\mathrm{H})^{\mathrm{k}}, \mathrm{k}=1,2, \ldots, \mathrm{n}-1$. Expanding $(\mathrm{J}-\mathrm{G})^{\mathrm{k}}$ and using Lemmas 3.3 and 3.4 we have

$$
\operatorname{tr}(J-G)^{k}=(-1)^{k}\left[\operatorname{tr} G^{k}-k \operatorname{tr} J G^{k-1}\right]+\phi_{k}(G)
$$

where $\phi_{k}(G)$ is a polynomial in $\left\{\operatorname{tr~} \mathrm{JG}^{\mathrm{r}}\right\}_{r=1}^{\mathrm{k}-2}$, with a corresponding expression for $\operatorname{tr}(\mathrm{J}-\mathrm{H})^{\mathrm{k}}$.

Since $\operatorname{tr} G^{k}=\mathrm{tr}^{k}$, the condition $\mathrm{tr} \mathrm{JG}^{\mathrm{r}}=\mathrm{tr} \mathrm{JH}^{\mathrm{r}}, \mathrm{r}=1,2, \ldots, \mathrm{k}-1$ gives us $\operatorname{tr}(J-G)^{k}=\operatorname{tr}(J-H)^{k}$, so that $\bar{G}$ and $\bar{H}$ are cospectral.

Conversely, the equation 3.7 can be solved uniquely for $\mathrm{tr} \mathrm{JG}^{\mathrm{k}-1}$, and by easy induction on $k$ we find that the condition $\operatorname{tr}(J-G)^{r}=\operatorname{tr}(J-H)^{r} \quad r=1,2, \ldots$ gives us $\operatorname{tr} \mathrm{JG}^{\mathrm{k}-1}=\operatorname{tr} \mathrm{JH}^{\mathrm{k}-1} \mathrm{k}=2,3, \ldots / /$

Corollary 3.8 [6]. If $\mathrm{G}$ and $\mathrm{H}$ are regular cospectral graphs, then $\overline{\mathrm{G}}$ and $\overline{\mathrm{H}}$ are cospectral.

Proof. If $G$ and $H$ are regular of degree $k$, then from [5] we have

$$
W_{G}(x)=W_{H}(x)=\frac{n k x}{1-k x} \quad \cdot / /
$$

Let $G_{1}$ and $G_{2}$ be two graphs. The join $G_{1}: G_{2}$ of $G_{1}$ and $G_{2}$ is formed by taking the disjoint union of $G_{1}$ and $G_{2}$ and joining each vertex of $G_{1}$ to each vertex of $G_{2}$.

Theorem 3.9. Let $\mathrm{G}$ and $\mathrm{H}$ be cospectral $\mathrm{n}$-graphs. Let $\mathrm{L}$ be any graph. Then $\overline{\mathrm{G}}$ and $\overline{\mathrm{H}}$ are cospectral iff $\mathrm{G}: \mathrm{L}$ and $\mathrm{H}: \mathrm{L}$ are cospectral.

Proof. Our original proof of this theorem was made obsolete by the following result of Cvetkovic [5], from which the result is immediate.

$$
(G: L)(\lambda)=(-1)^{l} G(\lambda) \bar{L}(-\lambda-1)+(-1)^{n} L(\lambda) \bar{G}(-\lambda-1)-(-1)^{n+l-\bar{G}(-\lambda-1) \bar{L}(-\lambda-1)}
$$

and similarly for $\mathrm{H}: \mathrm{L}$, where $\ell$ is the number of vertices of L.// 
4. GRAPHS COSPECTRAL TO THEIR OWN COMPLEMENTS

A graph is called sezf-complementary if it is isomorphic to its own complement. Such graphs are obviously cospectral to their own complements but it has been discovered, probably for the first time, that there are non-self-complementary graphs cospectral to their own complements. The smallest such graphs are shown in Figure 3 . of course, the complements of these graphs have the same property.
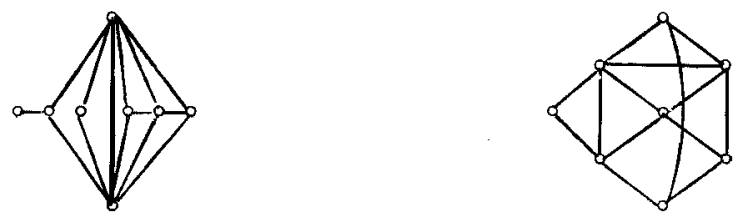

FIGURE 3

A list of all those graphs on 8 or 9 vertices which are cospectral to their own complements is given in Table 5. Those that are actually self-complementary are indicated by an asterisk. Otherwise, the complement of the graph must be added to the Table.

As indicated in the table by brackets, some cospectral families occur amongst graphs in this class. A particularly interesting family is that drawn in Figure 4.

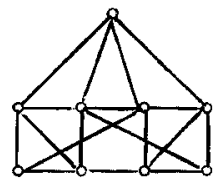

(a)

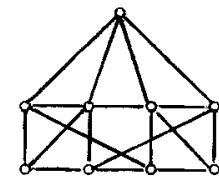

(b)

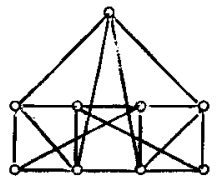

(c)

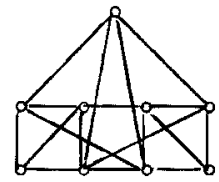

(d)

FIGURE 4

Graphs (a) and (b) are complements of each other, whereas graphs (c) and (d) are both self-complementary. All of these graphs have the polynomial $\lambda^{9}-18 \lambda^{7}-14 \lambda^{6}$ $+67 \lambda^{5}+60 \lambda^{4}-62 \lambda^{3}-46 \lambda^{2}+12 \lambda+8$. Graphs (a) and (b) have the additional property that their linegraphs are cospectral and have cospectral complements.

\section{COSPECTRAL GRAPHS WITH COSPECTRAL LINEGRAPHS}

In a similar fashion, one can ask for those cospectral graphs which have cospectral linegraphs. Such graphs are surprisingly uncomnon, at least on a small 
number of vertices. The only such families on 9 or fewer vertices are the four pairs of 9-graphs listed in Table 4. Note that the fourth pair is that drawn in Figure 4(a) and (b). Except for the thind pair, these pairs also have cospectral complements. However, their complements do not have cospectral linegraphs except for the fourth pair.

No simple characterization of such graphs seems to be known. However small amounts of information may be deduced, as for example in the following theorem.

Theorem 5.1. Let $\mathrm{G}$ and $\mathrm{H}$ be cospectral $\mathrm{n}$-graphs with cospectral linegraphs. Then if the degrees of the vertices of $\mathrm{G}$ and $\mathrm{H}$ are $\left\{\mathrm{g}_{i}\right\}_{1}^{\mathrm{n}}$ and $\left\{\mathrm{h}_{\mathrm{i}}\right\}_{1}^{\mathrm{n}}$ respectively,

$$
\sum_{i=1}^{n} g_{i}^{k}=\sum_{i=1}^{n} h_{i}^{k}, \quad k=1,2 \text { and } 3 \text {. }
$$

Proof. Let $B$ be the incidence matrix of $G,[9],[2]$. Then $B^{\mathrm{T}} \mathrm{B}=\mathrm{L}(\mathrm{G})+2 \mathrm{I}$, $\mathrm{BB}^{\mathrm{T}}=\mathrm{G}+\Gamma_{\mathrm{G}}$ where $\mathrm{I}$ is the identity matrix and $\Gamma_{G}=\operatorname{diag}\left(g_{1}, g_{2}, \ldots, g_{n}\right)$. Since $B \mathrm{~T}_{B}$ and $\mathrm{BB}^{\mathrm{T}}$ have the same non-zero eigenvalues [14], the polynomial of $L(G)$ is determined by the polynomial of $G+\Gamma_{G}$. Similarly for $H$.

From 3.2 the conditions of the theorem now become

$$
\operatorname{tr} G^{k}=\operatorname{tr} H^{k} ; \operatorname{tr}\left(G+\Gamma_{G}\right)^{k}=\operatorname{tr}\left(H+\Gamma_{H}\right)^{k} \quad k=1,2, \ldots
$$

The results now follow by expanding the second equation for the cases $k=1,2$ and $3 . / /$

Unfortunately, these conditions do not ensure that $G$ and $H$ have the same degree sequences, the third pair of graphs in Table 4 providing a counter-example.

6. COSPECTRAL BIPARTITE GRAPHS

A graph $G$ is said to be bipartite (also bicolorable) if the vertices of $G$ can be divided into two classes in such a way that no edge of $G$ joins two members of the same class. Such graphs are characterized by their spectrum, as shown in the following theorem ([7], quoted incorrectly in [21]).

Theorem 6.1. Let G be a graph. Then

(a) $G$ is bipartite iff to every eigenvalue $\lambda_{i}$ of $G$ there corresponds an eigenvalue $-\lambda_{i}$ of the same multipliaity. (Thus the polynomial of $G$ has all odd on alz even powers of $\lambda$.)

(b) If $G$ is connected and $r$ is the zargest positive eigenvalue of $G$, then $\mathrm{G}$ is bipartite iff $-\mathrm{r}$ is an eigenvalue of $\mathrm{G} . / /$

The distribution of cospectral families amongst the bipartite graphs is given in Table 10, where $b_{e}$ is the number of bipartite graphs with e edges. Since the actual values of $b_{e}$ seem to have only been published as fan as 6 vertices [12], all values of 
$e$ where $b_{e} \neq 0$ are included.

7. COSPECTRAL TREES

In the case of trees, the work of the preceding sections has been extended through 14 vertices. For this purpose, the trees themselves were machine generated, using a method similar to that described by Read [18].

The spectrum of a tree bears the following simple relationship to the structure of the tree. A k-matching of a tree $T$ is a set of $k$ mutually non-incident edges of $T$.

Theorem 7.1. Let $\mathrm{T}$ be a tree on $\mathrm{n}$ vertices. Then $\mathrm{I}$ has the polynomial

$$
\mathrm{T}(\lambda)=\sum_{k=0}^{\mathrm{D}} \mathrm{m}_{k} \lambda^{\mathrm{n}-2 \mathrm{k}} \text {, where } \mathrm{m}_{k}(k>0)
$$

is the number of $k$-matchings of $\mathrm{T}, \mathrm{m}_{0}=1$, and $\mathrm{p}$ is the integral part of $\frac{\mathrm{n}}{2}$.

Proof. First given by Sachs [19], and later independently by Mowshowitz [16].//

The smallest pair of cospectral trees, and the smallest foursome are shown in Figure 5.
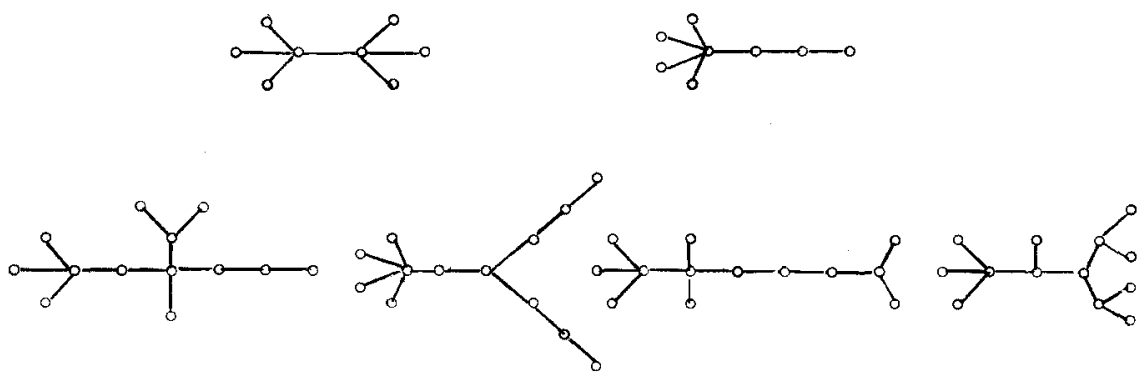

FIGURE 5

The distribution of cospectral families of trees from 8 through 14 vertices is given in Table 7 , where $t_{n}$ is the number of trees on $n$ vertices, and the other symbols are as in Table 1 . It is seen that the proportion of trees identified by their spectrum appears to be dropping rapidly, and may even fall below 50\% between 20 and 25 vertices.

The asymptotic value of this proportion has been discovered by Schwenk [20]:

Theorem 7.2. Let $\mathrm{P}_{\mathrm{n}}$ be the proportion of trees on $\mathrm{n}$ vertices which are identified (within the class of trees) by their spectrum.

Then $\mathrm{P}_{\mathrm{n}} \rightarrow 0$ as $\mathrm{n} \rightarrow \infty$. / 
8.

A 1-factor of a graph $G$ is a regular spanning subgraph of $G$ of degree one. Clearly a l-factor of a tree on $2 \mathrm{n}$ vertices corresponds to an n-matching. From 7.1 those trees with a l-factor are just those which have no zero eigenvalues.

The smallest examples of cospectral trees in this class are the two pairs shown in Figure 6.
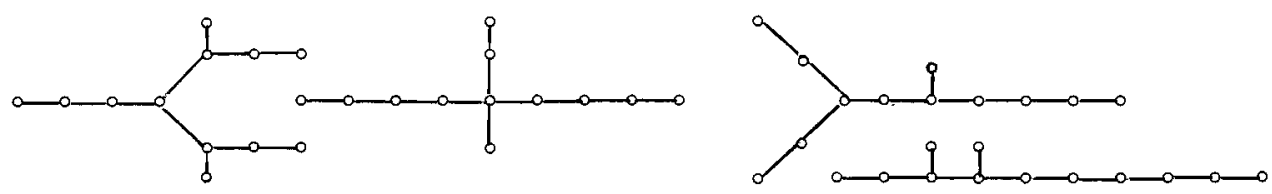

FIGURE 6

The frequency of occurrence of such families is given in Table 8 . As no enumeration of trees with a l-factor is known to the authors, all sizes from 2 through 14 vertices are included.

\section{COSPECTRAL TREES WITH COSPECTRAL COMPLEMENTS}

Let $G$ and $H$ be $n$ vertex and $m$ vertex rooted graphs, respectively. Define $G_{1}$ and $H_{1}$ to be the (unrooted) induced subgraphs of $G$ and $H$, respectively, formed by removing the root. The merge $\mathrm{G} \cdot \mathrm{H}$ of $\mathrm{G}$ and $\mathrm{H}$ is defined as the $\mathrm{n}+\mathrm{m}-\mathrm{l}$ vertex graph formed from the disjoint union of $G$ and $H$ by identifying the two roots.

Lemma 9.1.

$$
(G \cdot H)(\lambda)=G(\lambda) H_{1}(\lambda)+G_{1}(\lambda) H(\lambda)-\lambda G_{1}(\lambda) H_{1}(\lambda)
$$

where $G(\lambda)$ and $H(\lambda)$ are the polynomials of $G$ and $H$ considered as unrooted.

Proof. The determinant $|\lambda I-G \cdot H|$ can be written as

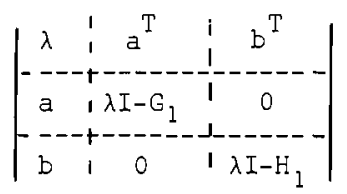

where $a$ and $b$ are vectors.

Since the determinant of a matrix is a linear function of its first row we have 


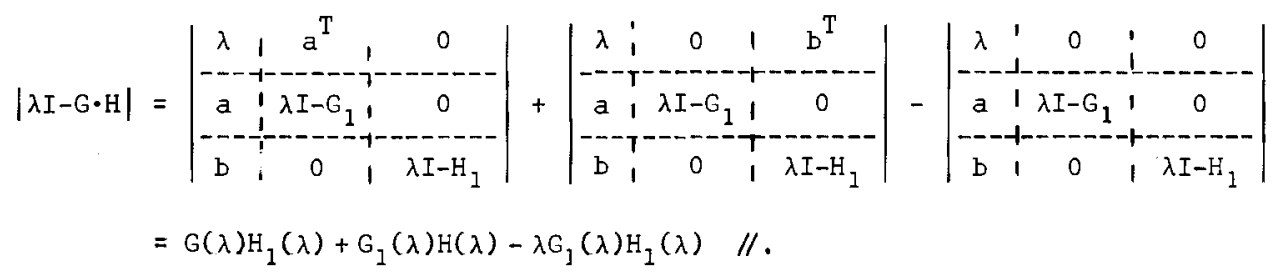

This Iemma has been previousiy given by Schwenk [20] for trees, in which case it is easily proved from Theorem 7.1.

Lemma 9.2. Define generating functions (see 53) as follows:

$$
\begin{aligned}
& \mathrm{W}_{G} \text { : all walks on } G \text {, } \\
& \mathrm{W}_{G}{ }^{\prime}: \text { all walks on } G \text { starting at the root, } \\
& \mathrm{W}_{G}{ }^{\prime} \text { : all walks on } G \text { starting and finishing at the root, }
\end{aligned}
$$

and similarly for $\mathrm{H}$ and $\mathrm{G} \cdot \mathrm{H}$. The coefficient of unity in all these functions is zero.

Then

$$
\begin{aligned}
& W_{G \cdot H}=W_{G}+W_{H}+\left(W_{G}{ }^{2} W_{H}^{\prime \prime}+2 W_{G}{ }^{\prime} W_{H}{ }^{\prime}+W_{G}{ }^{\prime \prime} W_{H}{ }^{2}\right) \sum_{r=0}^{\infty}\left(W_{G}{ }^{\prime \prime} W_{H}{ }^{\prime \prime}\right)^{r} \\
& =W_{G}+W_{H}+\frac{\left(W_{G}^{\prime 2} W_{H}^{\prime \prime}+2 W_{G}^{\prime} W_{H}^{\prime}+W_{G} " W_{H}^{\prime 2}\right)}{1-W_{G}{ }^{\prime \prime} W_{H}^{\prime \prime}}
\end{aligned}
$$

Proof. For walks on $G \cdot H$ of the form $e_{1}, e_{2}, \ldots, e_{k}$ where $e_{1}$ and $e_{k}$ are edges of $G$ we have the function

$$
W_{G}+W_{G}^{\prime} W_{H}^{\prime \prime} W_{G}^{\prime}+W_{G}{ }^{\prime} W_{H} " W_{G} " W_{H} " W_{G}^{\prime}+\ldots,
$$

and similarly when $e_{1}$ and $e_{k}$ are edges of $\mathrm{H}$.

If $e_{1}$ is an edge of $G$ and $e_{k}$ is an edge of $H$, or vice-versa, we find (in each case) the function

$$
W_{G}{ }^{\prime} W_{H}^{\prime}+W_{G}{ }^{\prime} W_{H}{ }^{\prime \prime} W_{G} " W_{H}{ }^{\prime}+W_{G}{ }^{\prime} W_{H}{ }^{\prime \prime} W_{G} " W_{H}{ }^{\prime \prime} W_{G} " W_{H}^{\prime}+\ldots
$$

Adding the four functions thus obtained gives the required result//.

A rooted tree $L$ is called a limb of a tree $T$ if for some rooted tree $R$ we have $\mathrm{T}=\mathrm{R} \cdot \mathrm{L}$.

Theorem 9.3. Let $\mathrm{G}$ be an arbitrary rooted graph. Let $\mathrm{S}$ and $\mathrm{T}$ be the rooted trees shown in Figure 7 . 

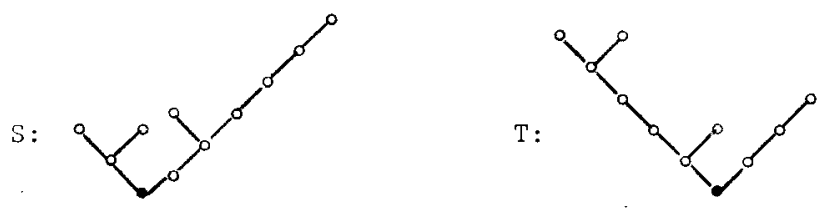

\section{FIGURE 7}

Then G.S and G.T are not isomorphic (if $G$ does not have an isolated root) but are cospectral and have cospectral complements.

Proof. (a) Since G.S has $P_{5}$ rooted at an end-vertex once more as a limb than has $G \cdot T$, it is not isomorphic to $G \cdot T$.

(b) $T$ and $S$ are isomorphic as unrooted trees, and $T_{1}$ and $S_{1}$ are isomorphic. So G.S and G.T are cospectral by Lemma 9.I.

(c) Direct computation from the adjacency matrices of $\mathrm{T}$ and $\mathrm{S}$ shows

that

$$
W_{T}^{\prime}(x)=W_{S}^{\prime}(x) \text { and } W_{T}^{\prime \prime}(x)=W_{S}^{\prime \prime}(x)
$$

Hence $W_{G} \cdot S=W_{G} \cdot T$ by Lemma 9.2 .

Hence $G \cdot S$ and $G \cdot T$ have cospectral complements, by $3.6 / /$.

The following result was first proved by Schwenk [20], who enumerated trees not containing a given limb. This enumeration has since been carried out more directly by McAvaney [15].

Lemma 9.4. Let $I_{1}$ and $I_{2}$ be rooted trees on the same number of vertices. Let $\mathrm{P}_{1}(\mathrm{n})$ and $\mathrm{P}_{2}(\mathrm{n})$ be respectively the number of (unrooted) trees on $\mathrm{n}$ vertices not containing $\mathrm{I}_{1}$ or $\mathrm{I}_{2}$ as a limb. Then $\mathrm{P}_{1}(\mathrm{n})=\mathrm{P}_{2}(\mathrm{n})$ for all $\mathrm{n}$.

Furthermore, if $t_{n}$ is the number of trees on $\mathrm{n}$ vertices,

$$
\frac{\mathrm{p}_{1}(\mathrm{n})}{\mathrm{t}_{\mathrm{n}}} \rightarrow 0 \text { as } \mathrm{n} \rightarrow \infty
$$

Theorem 9.5. Let $\mathrm{t}_{\mathrm{n}}$ be the number of trees on $\mathrm{n}$ vertices. Let $\mathrm{s}_{\mathrm{n}}$ be the number of trees on $\mathrm{n}$ vertices which are identified by their spectra together with the spectra of their complements.

Then $\frac{{ }^{s} \mathrm{n}}{\mathrm{t}_{\mathrm{n}}} \rightarrow 0$ as $\mathrm{n} \rightarrow \infty$.

Proof. By 9.3, any tree which can be written as $R \cdot S$ for some rooted tree $R$ is cospectral with cospectral complements to the (different) tree $R \cdot T$, where $S$ and $T$ are 
as in 9.3. By 9.4, the propontion of all trees which cannot be written in this form goes to zero as the size increases.//

Note 9.6. Theorem 9.3 can be generalized to show that for any rooted graphs $G$ and $H$ the two graphs shown schematically in Figure 8 are cospectral and have cospectral complements. In fact every pair of trees on 14 or fewer vertices which have this property fall into the class of graphs illustrated.
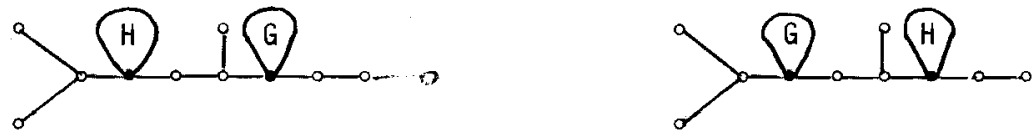

FIGURE 8

\section{COSPECTRAL TREES WITH COSPECTRAL LINEGRAPHS}

A search of the cospectral trees on up to 14 vertices has produced a small number of pairs which also have cospectral linegraphs. Specifically, there is $I$ pair on 11,1 pair on 12, 1 pair on 13 and 5 pairs on 14 vertices. Those on 11 vertices are shown in Figure 9.
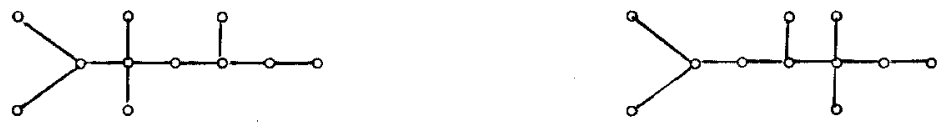

FIGURE 9

\section{COSPECTRAL FORESTS}

The smallest pair of cospectral forests are those shown in Figure 10.
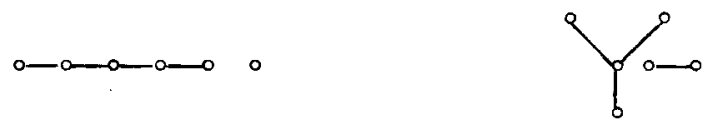

FIGURE 10

The distribution of such families for forests on up to 13 vertices is given in Table 9. It is seen that the proportion of forests determined by thein spectra drops more rapidly with increasing size than it does for trees. Extrapolation by hand suggests that the figure may drop below $50 \%$ by 16 vertices. 


\section{APPENDIX}

In order to reduce space requirements, a compact notation for graphs will be used. This is best illustrated by an example. Take the eight-vertex graph shown in Figure 1l, together with its adjacency matrix,

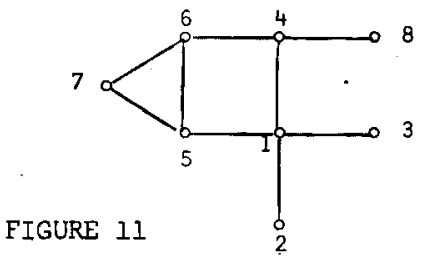

$$
\left[\begin{array}{llllllll}
0 & 1 & 1 & 1 & 1 & 0 & 0 & 0 \\
1 & 0 & 0 & 0 & 0 & 0 & 0 & 0 \\
1 & 0 & 0 & 0 & 0 & 0 & 0 & 0 \\
1 & 0 & 0 & 0 & 0 & 1 & 0 & 1 \\
1 & 0 & 0 & 0 & 0 & 1 & 1 & 0 \\
0 & 0 & 0 & 1 & 1 & 0 & 1 & 0 \\
0 & 0 & 0 & 0 & 1 & 1 & 0 & 0 \\
0 & 0 & 0 & 1 & 0 & 0 & 0 & 0
\end{array}\right]
$$

The lower triangle of the adjacency matrix, excluding the diagonal; is written down row by row as a binary integer:

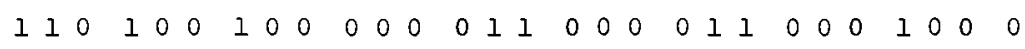

Zeros are added to the right-hand end if necessary to make the number of digits up to a multiple of three, and then the whole is written as an octal integer:

6440303040 . This notation is essentially the same as that employed by Baker, Dewdney and Szilard [1].

The following tables have been computer generated and checked carefully. Where overlap occurs with other existing tables [11], [13], [16], no discrepancies have been found. 
5 VERTICES:

$\begin{array}{ccccc}e & g_{e} & \phi_{2} & c_{e} & c_{e} / g_{e} \\ 4 & 6 & 1 & 2 & .333\end{array}$

TOTALS :

$\begin{array}{llll}34 & 1 & 2 & .059\end{array}$

7 VERTICES :

$\begin{array}{rrrrrl}\mathrm{e} & g_{\mathrm{e}} & \phi_{2} & \phi_{3} & c_{\mathrm{e}} & c_{\mathrm{e}} / g_{\mathrm{e}} \\ 4 & 10 & 2 & & 4 & .400 \\ 5 & 21 & 0 & 1 & 3 & .143 \\ 6 & 41 & 7 & & 14 & .341 \\ 7 & 65 & 4 & & 8 & .123 \\ 8 & 97 & 9 & & 18 & .186 \\ 9 & 131 & 6 & & 12 & .092 \\ 10 & 148 & 8 & & 16 & .108 \\ 11 & 148 & 5 & 1 & 13 & .088 \\ 12 & 131 & 6 & & 12 & .092 \\ 13 & 97 & 2 & & 4 & .041 \\ 14 & 65 & 1 & & 2 & .031 \\ 15 & 41 & 1 & & 2 & .049 \\ 16 & 21 & 1 & & 2 & .095\end{array}$

TOTALS:

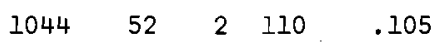

6 VERTICES:

$\begin{array}{ccccc}e & g_{e} & \phi_{2} & c_{e} & c_{e} / g_{e} \\ 4 & 9 & 2 & 2 & .444 \\ 5 & 15 & 1 & 2 & .133 \\ 6 & 21 & 1 & 2 & .095 \\ 7 & 24 & 1 & 2 & .083\end{array}$

TOTALS :

$\begin{array}{llll}156 & 5 & 10 & .064\end{array}$

8 VERTICES:

$\begin{array}{rrrrrrr}e & g_{e} & \phi_{2} & \phi_{3} & \phi_{4} & c_{e} & c_{e} / g_{e} \\ 4 & 11 & 2 & & & 4 & .364 \\ 5 & 24 & 1 & 1 & & 5 & .208 \\ 6 & 56 & 7 & 1 & 1 & 21 & .375 \\ 7 & 115 & 13 & & & 26 & .226 \\ 8 & 221 & 25 & 3 & 1 & 63 & .285 \\ 9 & 402 & 28 & 4 & & 68 & .169 \\ 10 & 663 & 67 & 10 & & 164 & .247 \\ 11 & 980 & 66 & 4 & 1 & 148 & .151 \\ 12 & 1312 & 97 & 7 & 1 & 219 & .167 \\ 13 & 1557 & 105 & 3 & 1 & 223 & .143 \\ 14 & 1646 & 99 & 7 & & 219 & .133 \\ 15 & 1557 & 102 & 2 & & 210 & .135 \\ 16 & 1312 & 68 & 5 & & 151 & .115 \\ 17 & 980 & 42 & 1 & 1 & 91 & .093 \\ 18 & 663 & 29 & 2 & & 64 & .097 \\ 19 & 402 & 15 & & & 30 & .075 \\ 20 & 221 & 2 & 2 & & 10 & .045 \\ 21 & 115 & 2 & & & 4 & .035 \\ 22 & 56 & 1 & & & 2 & .036\end{array}$

TOTALS:

$\begin{array}{llllll}12346 & 771 & 52 & 6 & 1722 & .139\end{array}$

TABLE 1. COSPECTRAL GRAPHS 
9 VERTICES:

\begin{tabular}{|c|c|c|c|c|c|c|c|c|c|c|c|c|}
\hline e & $g_{e}$ & $\phi_{2}$ & $\phi_{3}$ & $\phi_{4}$ & $\phi_{5}$ & $\phi_{6}$ & $\phi_{7}$ & $\phi_{8}$ & $\phi_{9}$ & $\phi_{10}$ & $c_{e}$ & $c_{e} / g_{e}$ \\
\hline 4 & 11 & 2 & & & & & & & & & 4 & .364 \\
\hline 5 & 25 & 1 & $I$ & & & & & & & & 5 & .200 \\
\hline 6 & 63 & 8 & 1 & 0 & 1 & & & & & & 24 & .381 \\
\hline 7 & 148 & 19 & 3 & 1 & & & & & & & 51 & .345 \\
\hline 8 & 345 & 50 & 5 & 4 & 1 & & & & & & 136 & .394 \\
\hline 9 & 771 & 75 & 11 & 4 & 1 & & & & & & 204 & .265 \\
\hline 10 & 1637 & 169 & 41 & 10 & 1 & 1 & & & & & 512 & .313 \\
\hline 11 & 3252 & 305 & 35 & 5 & 1 & & & & & & 740 & .228 \\
\hline 12 & 5995 & 494 & 109 & 29 & 2 & 3 & & & & & 1419 & .237 \\
\hline 13 & 10120 & 840 & 89 & 22 & 6 & & & & & & 2065 & .204 \\
\hline 14 & 15615 & 1273 & 167 & 37 & 10 & 5 & $I$ & & & & 3282 & .210 \\
\hline 15 & 21933 & 1833 & 149 & 49 & 2 & 2 & & & & & 4331 & .197 \\
\hline 16 & 27987 & 2173 & 261 & 57 & 18 & 8 & 0 & 1 & 0 & 1 & 5513 & .197 \\
\hline 17 & 32403 & 2742 & 179 & 75 & 2 & 2 & & & & & 6338 & .196 \\
\hline 18 & 34040 & 2615 & 257 & 72 & 17 & 5 & & & & & 6404 & .188 \\
\hline 19 & 32403 & 2626 & 154 & 66 & 0 & 2 & & & & & 5990 & .185 \\
\hline 20 & 27987 & 1979 & 205 & 54 & 20 & 4 & 0 & 1 & 0 & 1 & 4931 & .176 \\
\hline $2 I$ & 21933 & 1600 & 110 & 36 & 3 & 1 & & & & & 3695 & .268 \\
\hline 22 & 15615 & 983 & 118 & 20 & 8 & 3 & & & & & 2458 & .157 \\
\hline 23 & 10120 & 652 & 41 & 17 & 1 & & & & & & 1500 & .248 \\
\hline 24 & 5995 & 296 & 58 & 3 & 1 & 1 & & & & & 789 & .132 \\
\hline 25 & 3252 & 286 & 6 & 0 & 1 & & & & & & 395 & .121 \\
\hline 26 & 2637 & 63 & 12 & & & & & & & & 162 & .099 \\
\hline 27 & 771 & 30 & 1 & & & & & & & & 63 & .082 \\
\hline 28 & 345 & 6 & 2 & & & & & & & & 18 & .052 \\
\hline 29 & 148 & 4 & & & & & & & & & 8 & .054 \\
\hline 30 & 63 & 1 & & & & & & & & & 2 & .032 \\
\hline
\end{tabular}

TOTALS:

$\begin{array}{lllllllllll}274668 & 21025 & 2015 & 551 & 95 & 37 & 1 & 2 & 0 & 2 & 51039\end{array}$

TABLE 1. COSPECTRAL GRAPHS (cont.) 


\begin{tabular}{|c|c|c|c|c|c|}
\hline 6440 & 3600 & & $\begin{array}{l}7654001 \\
7651400\end{array}$ & $\begin{array}{l}7741100 \\
7741010\end{array}$ & * \\
\hline 64400 & 36000 & & 7652001 & 7651004 & \\
\hline $\begin{array}{l}24410 \\
64210\end{array}$ & $\begin{array}{l}34200 \\
36001\end{array}$ & & $\begin{array}{l}7445210 \\
7610302\end{array}$ & $\begin{array}{l}7641003 \\
7640403\end{array}$ & $*$ \\
\hline 74440 & 76001 & & 7445011 & 3651202 & $*$ \\
\hline 74460 & 76102 & & $\begin{array}{l}7760402 \\
7742140\end{array}$ & $\begin{array}{l}7762001 \\
7760101\end{array}$ & \\
\hline 6440000 & 3600000 & & 7663040 & 6771400 & \\
\hline 2441000 & 3420000 & & 7656020 & 7662440 & \\
\hline 6421000 & 2442020 & 3600100 & 7655001 & 7662041 & \\
\hline 3660000 & 6442040 & & 7660405 & 7650301 & $*$ \\
\hline 6442020 & 3642000 & & 7445602 & 7651005 & $*$ \\
\hline 3640100 & 6441020 & & 6362444 & 6651430 & $*$ \\
\hline 6441010 & 3640001 & & 7762500 & 7772040 & \\
\hline 3441020 & 3600102 & & 7762140 & 7762402 & \\
\hline 6420404 & 3420300 & $*$ & 7655440 & 7762021 & \\
\hline 7444000 & 7600100 & & $7655401 *$ & 7656041 & $7760405 *$ \\
\hline 3660001 & 3642040 & & 7650341 & 6655430 & $*$ \\
\hline 7446000 & 7610200 & & 6636430 & 7640742 & $*$ \\
\hline 7424400 & 7441020 & & 7762444 & 7762430 & $*$ \\
\hline 7420300 & 7420404 & is & 7772021 & 7762504 & \\
\hline 7642040 & 7616000 & & 7672604 & 7763042 & \\
\hline 7642020 & 7641100 & & 7652243 & 7760603 & \\
\hline 7614001 & 7446040 & & 7465614 & 7662407 & $*$ \\
\hline 7541020 & 7642002 & & 3673214 & 7652223 & $*$ \\
\hline 6651400 & 7640404 & $*$ & 7762544 & 7762530 & $*$ \\
\hline 7446020 & 7612002 & & 7762407 & 7655603 & $*$ \\
\hline 3651200 & 6660410 & & 7762546 & 7672634 & $*$ \\
\hline 7445020 & 7640101 & & 7655770 & 7762547 & $*$ \\
\hline 7440203 & 4651410 & $*$ & 7777603 & 7777470 & \\
\hline
\end{tabular}

761644106510 746262406450 363646144011 756222120451 743704411430 743234426042 761614130430 365166244011 365233124012 761613006604

TABLE 3. 10 COSP. 9-GRAPHS

714624020500
724622010600
765466121011
765466023006
744725236006
770315226070
753222342524
753212545112

TABLE 4. COSP. GRAPHS WITH COSP. LINEGRAPHS

$\begin{array}{lll}7773240200 * & 677546161040 & 765546144015 \\ 7773060010 & 771746442110 * & 376252650121 * \\ 7763014140 * & 767642550120 * & 770315226070 * \\ 7762424140 * & 776252650120 * & 367645025122 * \\ 3773240204 * & 776315244044 & 765164660015 \\ 7651350240 * & 775613160120 & {[765131344540} \\ 7651344300 * & 767642544140 * & 376252644141 * \\ 7651407440 & 776252644140 * & 367644430524 * \\ 3663114144 * & 775714120502 & 765062362026 \\ 6351246310 * & 777320340502 * & 753212544514 * \\ 3652426250 * & 377326340101 * & 377320340503 * \\ 3652231260 * & 766263344110 * & 353726241103 * \\ & 756711544140 & 753212545112 \\ 777746440100 *[766263342120 * & 754152643122 * \\ 777326340100 * & 765163443210 & 753222344514 * \\ 777346460001 & 577325211201 & 765130350522 * \\ 777706014060 * & 765234631140 & 353726240505 * \\ 777705024060 * & 376252750120 & 754542343122 * \\ 776657041100 & 766263242121 & 765130344542 * \\ 757562560200 & 367706114061 * & 227645025526 * \\ 773364242030 & 770316216070 * & 354542343123 * \\ 777135140201 & 637642445142 * & 376212211427 * \\ 377746440101 * & 766263412031 * & 365223126017 *\end{array}$

TABLE 5. GRAPHS COSPECTRAL TO THEIR OWN COMPLEMENTS *Indicates self-complementary [ Indicates cospectrality 
7 VERTICES :

$\begin{array}{rcccc}e & g_{e} & \phi_{2} & c_{e} & c_{e} / g_{e} \\ 6 & 41 & 1 & 2 & .049 \\ 7 & 65 & 1 & 2 & .031 \\ 8 & 97 & 2 & 4 & .041 \\ 9 & 131 & 3 & 6 & .046 \\ 10 & 148 & 3 & 6 & .041\end{array}$

TOTALS:

$1044 \quad 20 \quad 40 \quad .038$
8 VERTICES :

\begin{tabular}{|c|c|c|c|c|c|c|}
\hline e & $g_{e}$ & $\phi_{2}$ & $\phi_{3}$ & $\phi_{4}$ & $\mathrm{c}_{\mathrm{e}}$ & $c_{e} / g_{e}$ \\
\hline 6 & 56 & 1 & & & 2 & .036 \\
\hline 7 & 115 & 2 & & & 4 & .035 \\
\hline 8 & 221 & 2 & 2 & & 10 & .045 \\
\hline 9 & 402 & 15 & & & 30 & .075 \\
\hline 10 & 663 & 26 & 1 & & 55 & .083 \\
\hline 11 & 980 & 40 & 1 & 1 & 87 & .089 \\
\hline 12 & 1312 & 59 & 5 & & 133 & 101 \\
\hline 13 & 1557 & 85 & 1 & & 173 & .111 \\
\hline 14 & 1646 & 86 & 2 & & 178 & .108 \\
\hline \multicolumn{7}{|c|}{ TOTALS: } \\
\hline & 12346 & 546 & 22 & 2 & 1166 & .094 \\
\hline
\end{tabular}

9 VERTICES;

\begin{tabular}{|c|c|c|c|c|c|c|c|c|c|c|c|c|}
\hline e & $g_{e}$ & $\phi_{2}$ & $\phi_{3}$ & $\phi_{4}$ & $\phi_{5}$ & $\phi_{6}$ & $\phi_{7}$ & $\phi_{8}$ & $\phi_{g}$ & $\phi_{10}$ & $c_{e}$ & $c_{e} / g_{e}$ \\
\hline 6 & 63 & 1 & & & & & & & & & 2 & .032 \\
\hline 7 & 148 & 3 & & & & & & & & & 6 & .041 \\
\hline 8 & 345 & 6 & 2 & & & & & & & & 18 & .052 \\
\hline 9 & 771 & 29 & 1 & & & & & & & & 61 & .079 \\
\hline 10 & 1637 & 61 & 12 & & & & & & & & 158 & .097 \\
\hline 11 & 3252 & 180 & 6 & 0 & 1 & & & & & & 383 & .118 \\
\hline 12 & 5995 & 284 & 58 & 3 & 2 & & & & & & 764 & .127 \\
\hline 13 & 10120 & 646 & 36 & 16 & 1 & & & & & & 1469 & .145 \\
\hline 14 & 15615 & 935 & 113 & 20 & 7 & 3 & & & & & 2342 & .150 \\
\hline 15 & 21933 & 1550 & 99 & 36 & 2 & 1 & & & & & 3557 & .162 \\
\hline 16 & 27987 & 1861 & 203 & 44 & 15 & 4 & 0 & 1 & 0 & 1 & 4624 & .165 \\
\hline 17 & 32403 & 2482 & 133 & 61 & 0 & 2 & & & & & 5619 & .173 \\
\hline 18 & 34040 & 2401 & 224 & 63 & 11 & 4 & & & & & 5805 & .17 \\
\hline \multicolumn{13}{|c|}{ TOTALS: } \\
\hline & 274668 & 18477 & 1550 & 423 & 67 & 24 & 0 & 2 & 0 & 2 & 43811 & 16 \\
\hline
\end{tabular}

TABLE 6. COSPECTRAL GRAPHS WITH COSPECTRAL COMPLEMENTS

\begin{tabular}{rrrrrrr}
$\mathrm{n}$ & $\mathrm{t}_{\mathrm{n}}$ & $\phi_{2}$ & $\phi_{3}$ & $\phi_{4}$ & \multicolumn{1}{c}{$\mathrm{c}_{\mathrm{n}}$} & $\mathrm{c}_{\mathrm{n}} / \mathrm{t}_{\mathrm{n}}$ \\
8 & 23 & 1 & & & 2 & .087 \\
9 & 47 & 5 & & & 10 & .213 \\
10 & 106 & 4 & & & 8 & .075 \\
11 & 235 & 27 & 2 & & 60 & .255 \\
12 & 551 & 49 & 7 & & 119 & .216 \\
13 & 1301 & 162 & 29 & 1 & 415 & .319 \\
14 & 3159 & 349 & 36 & 5 & 826 & .261
\end{tabular}

TABLE 7. COSPECTRAL TREES

\begin{tabular}{rrrrc}
$\mathrm{n}$ & \multicolumn{1}{c}{$\mathrm{t}_{\mathrm{n}}$} & $\phi_{2}$ & $\mathrm{c}_{\mathrm{n}}$ & $\mathrm{c}_{\mathrm{n}} / \mathrm{t}_{\mathrm{n}}$ \\
2 & 1 & 0 & 0 & $\cdots$ \\
4 & 1 & 0 & 0 & $\cdots$ \\
6 & 2 & 0 & 0 & $\cdots$ \\
8 & 5 & 0 & 0 & $\cdots$ \\
10 & 15 & 0 & 0 & $\cdots$ \\
12 & 49 & 2 & 4 & .082 \\
14 & 180 & 12 & 24 & .133
\end{tabular}

TABLE 8. TREES WITH I-FACTORS 


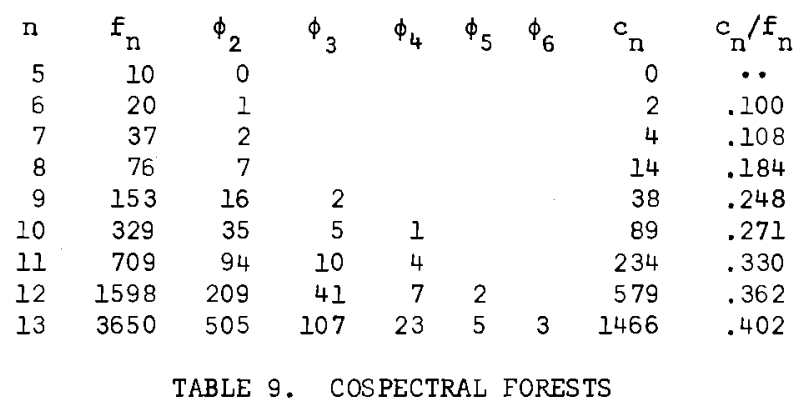

5 VERTICES :

$\begin{array}{ccccc}\mathrm{e} & \mathrm{b}_{\mathrm{e}} & \phi_{2} & \mathrm{c}_{\mathrm{e}} & \mathrm{c}_{\mathrm{e}} / \mathrm{b}_{\mathrm{e}} \\ 1 & 1 & 0 & 0 & \cdots \\ 2 & 2 & 0 & 0 & \cdots \\ 3 & 3 & 0 & 0 & \cdots \\ 4 & 4 & 1 & 2 & .500 \\ 5 & 1 & 0 & 0 & \cdots \\ 6 & 1 & 0 & 0 & \cdots\end{array}$

TOTALS:

$\begin{array}{llll}12 & 1 & 2 & .167\end{array}$
6 VERTICES :

$\begin{array}{ccccc}\mathrm{e} & \mathrm{b}_{\mathrm{e}} & \phi_{2} & \mathrm{c}_{\mathrm{e}} & \mathrm{c}_{\mathrm{e}} / \mathrm{b}_{\mathrm{e}} \\ 1 & 1 & 0 & 0 & \cdots \\ 2 & 2 & 0 & 0 & \cdots \\ 3 & 4 & 0 & 0 & \cdots \\ 4 & 7 & 2 & 4 & .571 \\ 5 & 8 & 1 & 2 & .250 \\ 6 & 6 & 0 & 0 & \cdots \\ 7 & 3 & 0 & 0 & \cdots \\ 8 & 2 & 0 & 0 & \cdots \\ 9 & 1 & 0 & 0 & \cdots\end{array}$

TOTALS :

$\begin{array}{llll}34 & 3 & 6 & .176\end{array}$

\section{VERTICES :}

$\begin{array}{rrrrrc}\mathrm{e} & \mathrm{b}_{\mathrm{e}} & \phi_{2} & \phi_{3} & \mathrm{c}_{\mathrm{e}} & \mathrm{c}_{\mathrm{e}} / \mathrm{b} \\ 1 & 1 & 0 & & 0 & \ldots \\ 2 & 2 & 0 & & 0 & \cdots \\ 3 & 4 & 0 & & 0 & \cdots \\ 4 & 8 & 2 & & 4 & .500 \\ 5 & 13 & 0 & 1 & 3 & .231 \\ 6 & 19 & 6 & & 12 & .632 \\ 7 & 14 & 1 & & 2 & .143 \\ 8 & 13 & 0 & & 0 & \cdots \\ 9 & 7 & 0 & & 0 & \cdots \\ 10 & 4 & 0 & & 0 & \cdots \\ 11 & 1 & 0 & & 0 & \cdots \\ 12 & 1 & 0 & & 0 & \cdots\end{array}$

TOTALS:

$$
\begin{array}{lllll}
87 & 9 & 1 & 21 & .241
\end{array}
$$

8 VERTICES :

$\begin{array}{rrrrrrc}\mathrm{e} & \mathrm{b}_{\mathrm{e}} & \phi_{2} & \phi_{3} & \phi_{4} & c_{\mathrm{e}} & c_{\mathrm{e}} / \mathrm{b}_{\mathrm{e}} \\ 1 & 1 & 0 & & & 0 & \cdots \\ 2 & 2 & 0 & & & 0 & \cdots \\ 3 & 4 & 0 & & & 0 & \cdots \\ 4 & 9 & 2 & & & 4 & .444 \\ 5 & 16 & 1 & 1 & & 5 & .313 \\ 6 & 32 & 6 & 1 & 1 & 19 & .594 \\ 7 & 45 & 7 & & & 14 & .311 \\ 8 & 52 & 5 & 2 & & 16 & .308 \\ 9 & 48 & 1 & 1 & & 5 & .104 \\ 10 & 40 & 5 & & & 10 & .250 \\ 11 & 24 & 0 & & & 0 & \cdots \\ 12 & 16 & 1 & & & 2 & .125 \\ 13 & 7 & 0 & & & 0 & \cdots \\ 14 & 3 & 0 & & & 0 & \cdots \\ 15 & 2 & 0 & & & 0 & \cdots \\ 16 & 1 & 0 & & & 0 & \cdots\end{array}$

TOTALS :

$\begin{array}{llllll}302 & 28 & 5 & 1 & 75 & .248\end{array}$ 
9 VERTICES :

$\begin{array}{rrrrrrrrc}\mathrm{e} & \mathrm{b}_{\mathrm{e}} & \phi_{2} & \phi_{3} & \phi_{4} & \phi_{5} & \phi_{6} & c_{\mathrm{e}} & c_{\mathrm{e}} / \mathrm{b} e \\ 1 & 1 & 0 & & & & & 0 & \ldots \\ 2 & 2 & 0 & & & & & 0 & \cdots \\ 3 & 4 & 0 & & & & & 0 & \cdots \\ 4 & 9 & 2 & & & & & 4 & .444 \\ 5 & 17 & 1 & 1 & & & & 5 & .294 \\ 6 & 38 & 7 & 1 & 0 & 1 & & 22 & .579 \\ 7 & 70 & 12 & 3 & 1 & & & 37 & .529 \\ 8 & 120 & 23 & 4 & 3 & 1 & & 75 & .625 \\ 9 & 150 & 23 & 2 & 2 & 1 & & 65 & .433 \\ 10 & 179 & 22 & 6 & 3 & 0 & 1 & 80 & .447 \\ 11 & 164 & 23 & 2 & & & & 52 & .317 \\ 12 & 143 & 17 & 4 & & & & 46 & .322 \\ 13 & 94 & 7 & & & & & 14 & .149 \\ 14 & 63 & 3 & 1 & & & & 9 & .143 \\ 15 & 32 & 1 & & & & & 2 & .063 \\ 16 & 19 & 0 & & & & & 0 & \ldots \\ 17 & 7 & 0 & & & & & 0 & \cdots \\ 18 & 4 & 0 & & & & & 0 & \ldots \\ 19 & 1 & 0 & & & & & 0 & \ldots \\ 10 & 1 & 0 & & & & & 0 & \cdots\end{array}$

TOTALS:

$\begin{array}{llllllll}1118 & 141 & 24 & 9 & 3 & 1 & 411 & .368\end{array}$

TABLE 10. COSPECTRAL BIPARTITE GRAPHS (cont.) 


\section{Added in proof:}

Recent computational work has extended the results of Table 7 to include trees on up to 18 vertices. The extended version of the table is presented below.

\begin{tabular}{|c|c|c|c|c|c|c|c|c|c|c|}
\hline $\mathrm{n}$ & $t_{n}$ & $\phi_{2}$ & $\phi_{3}$ & $\phi_{4}$ & $\phi_{5}$ & $\phi_{6}$ & $\phi_{7}$ & $\phi_{8}$ & $c_{n}$ & $c_{n} / t_{n}$ \\
\hline 8 & 23 & 1 & & & & & & & 2 & .087 \\
\hline 9 & 47 & 5 & & & & & & & 10 & .213 \\
\hline 10 & 106 & 4 & & & & & & & 8 & .075 \\
\hline 11 & 235 & 27 & 2 & & & & & & 60 & .255 \\
\hline 12 & 551 & 49 & 7 & & & & & & 119 & .216 \\
\hline 13 & 1301 & 162 & 29 & 1 & & & & & 415 & .319 \\
\hline 14 & 3159 & 349 & 36 & 5 & & & & & 826 & .261 \\
\hline 15 & 7741 & 960 & 145 & 20 & 7 & & & & 2470 & .319 \\
\hline 16 & 19320 & 2028 & 326 & 44 & 6 & 1 & & & 5246 & .272 \\
\hline 17 & 48629 & 5343 & 985 & 221 & 58 & 18 & 3 & & 14944 & .307 \\
\hline 18 & 123867 & 12163 & 1935 & 405 & 88 & 16 & 4 & 4 & 32347 & .261 \\
\hline
\end{tabular}

\section{EXTENDED TABLE 7. COSPECTRAL TREES}

It is interesting to note that the proportion $c_{n} / t_{n}$ tends to be larger for odd $\mathrm{n}$ than for even $\mathrm{n}$, and that the last few values appear to be dropping, despite the fact that $c_{n} / t_{n}$ tends to 1 for large $n$ (by 7.2). We have no explanation for these phenomena.

\section{REFERENCES}

[1] H. Baker, A. Dewdney and A. Szilard, Generating the nine-point graphs, Math. Comp. 28, $127(1974), 833-838$.

[2] M. Behzad and G. Chartrand, Introduction to the Theory of Graphs. (Allyn and Bacon, 1971.)

[3] I. Berezin and N. Zhidkov, Computing Methods, Vol. 2. (Pergamon 1965.)

[4] L. Collatz and U. Singowitz, Spektren endlicher Grafen. Abh. Math. Sem. Univ. Hamburg 21 (1957), 63-77.

[5] D. Cvetković, Graphs and their spectra, Publ. EZlek. Fak. Univ. Beograd: Ser. Mat. Fiz. $354(1971), 1-50$.

[6] H. Finck and G. Grohman, Vollständiges Produkt, chromatische Zahl und charakteristisches Polynom regularer Graphen (I), Wiss. Z. Techn. Hochsch. Ilmenau 11 (1965), 1-3. 
[7] F. Gantmacher, Applications of the Theory of Matrices, Vol. 2. (Chelsea, 1959.)

[8] C. Godsil and B. McKay, Products of graphs and their spectra, this volume.

[9] F. Harary, Graph Theory. (Addison-Wesley, 1969.)

[10] F. Harary and E. Palmer, Graphical enumeration. (Academic Press, 1973).

[1l] F. Harary, C. King, A. Mowshowitz and R. Read, Cospectral graphs and digraphs, BulZ. London Math. Soc. 3 (1971), 321-328.

[12] F. Harary and G. Prins, Enumeration of bicolourable graphs, Canad. J. Math. 15 (1963), 237-248.

[13] C. King, Characteristic polynomials of 7-node graphs, Scientific Rept., Univ. of the West Indies/CC 6 (AFOSR project 1026-66).

[14] M. Marcus and H. Minc, A Survey of Matrix Theory and Matrix Inequalities. (Allyn and Bacon, 1964.)

[15] K. McAvaney, Annote on limbless trees, Bull. Austral. Math. Soc. Il (1974), 381-384.

[16] A. Mowshowitz, The characteristic polynomial of a graph, J. Comb. Th. 12(B), (1972), 177-193.

[17] R. Read, Teaching graph theory to a computer, Recent progress in Combinatorics Proc. Third Waterloo Conf. Combinatorics. (Academic Press, 1968) 161-174.

[18] R. Read, The coding of various kinds of unlabelled trees, Graph Theory and Computing (ed. R. Read) (Academic Press, 1972) 153-183.

[19] H. Sachs, Beziehungen zwischen den in einem Graphen enthaltenen Kreisen und seinem charakteristischen Polynom., Publ. Math. Debrecen 9 (1962), 270-288.

[20] A. Schwenk, Almost all trees are cospectral. New Directions in the Theory of Grophs. (Proc. Third Ann Arbor Conf., Michigan) (Academic Press, 1973), 275-307.

[21] R. Wilson, on the adjacency matrix of a graph, Combinatorics. (Proc. Conf. Comb. Maths., Oxford) (Inst. Math. Appl., 1972), 295-321.

Department of Mathematics,

University of Melbourne,

Parkville, VIC. 